\title{
ABSORCIÓN Y CONCENTRACIÓN DE NITRÓGENO, FÓSFORO Y POTASIO EN SACHA INCHI (Plukenetia volubilis L.) EN SUELOS ÁCIDOS, SAN MARTÍN, PERÚ
}

Rafael Ananías BALTA CRISÓLOGO ${ }^{1,2}$ Ángel Martín RODRÍGUEZ DEL CASTILLO' Roger GUERRERO ABAD ${ }^{1,3}$, Danter CACHIQUE ${ }^{1}$, Edín ALVA PLASENCIA ${ }^{2}$, Luis ARÉVALO LÓPEZ ${ }^{1}$, Oscar $\mathrm{LOLI}^{4}$

1 Instituto de Investigaciones de la Amazonía Peruana (IIAP). Programa de Investigación en Biodiversidad Amazónica (PIBA) / Programa de Investigación en Manejo Integral del Bosque y Servicios Ambientales (PROBOSQUES). Tarapoto, San Martín, Perú. rafael.baltac@gmail.com; angelmartinrdc@gmail.com.

2 Universidad Nacional de Cajamarca (UNC). Facultad de Ciencias Agrarias. Cajamarca, Perú.

3 Universidad Nacional de San Martín (UNSM). Facultad de Ciencias Agrarias. Departamento Académico Agrosilvo Pastoril. Tarapoto, San Martín, Perú.

4 Universidad Nacional Agraria La Molina (UNALM). Facultad de Agronomía, Departamento Académico de Suelos. La Molina, Lima, Perú.

\section{RESUMEN}

El objetivo del presente trabajo fue determinar la absorción y la concentración de nitrógeno ( $N$ ), fósforo (P) y potasio (K) en el cultivo de sacha inchi (Plukenetia volubilis L.), con la finalidad de mejorar la eficiencia en la aplicación de fertilizantes. Los tratamientos evaluados fueron: $\mathrm{T}_{1}: 16-23-21 \mathrm{~kg} \mathrm{~N}, \mathrm{P}_{2} \mathrm{O}_{5}, \mathrm{~K}_{2} \mathrm{O}_{\text {ha }}{ }^{-1}, \mathrm{~T}_{2}: 8$

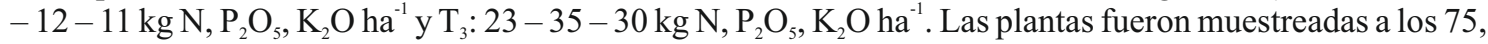
112,150 y 225 días después de la siembra. La concentración de N, P y K en cada organismo (raíz, tallo, hoja y fruto) fue evaluada usando el peso fresco, peso seco y área foliar. Los resultados nos indican que el tratamiento con mayor dosis de fertilizantes presentó los mejores rendimientos en materia seca, que fueron estadísticamente superiores a los producidos en el tratamiento 2 y 1 respectivamente. Así mismo, se encontró que el elemento acumulado en una mayor cantidad fue el nitrógeno, con $72.06 \mathrm{~kg} \mathrm{~N} \mathrm{ha}^{-1}$, el potasio alcanzó una concentración de $64.39 \mathrm{~kg} \mathrm{~K} \mathrm{ha}^{-1}$ y el fósforo fue el elemento que se acumuló en menor cantidad, registrando, $14.36 \mathrm{~kg} \mathrm{P} \mathrm{ha}^{-1}$.Las mayores concentraciones fueron obtenidas a los 225 días después de la siembra.

PALABRAS CLAVE: Plukenetia volubilis, nutrición mineral, absorción, nitrógeno, fósforo, potasio.

\section{ABSORPTION AND CONCENTRATION OF NITROGEN, PHOSPHORUS AND POTASSIUM IN SACHA INCHI (Plukenetia volubilis L.) PLANTED IN ACID SOILS OF SAN MARTIN, PERU.}

\begin{abstract}
The aim of this study was to determine the absorption and concentration of nitrogen $(\mathrm{N})$, phosphorus $(\mathrm{P})$ and potassium $(\mathrm{K})$ in Sacha inchi (Plukenetia volubilis L.) plantations, in order to improve the efficiency of applied fertilizers. The treatments were: $\mathrm{T}_{1}: 16-23-21 \mathrm{~kg} \mathrm{~N}, \mathrm{P}_{2} \mathrm{O}_{5}, \mathrm{~K}_{2} \mathrm{O} \mathrm{ha}^{-1}, \mathrm{~T}_{2}: 8-12-11 \mathrm{~kg} \mathrm{~N}, \mathrm{P}_{2} \mathrm{O}_{5}, \mathrm{~K}_{2} \mathrm{O}$ ha ${ }^{1} \mathrm{y} \mathrm{T}_{3}: 23-35-30 \mathrm{~kg} \mathrm{~N}, \mathrm{P}_{2} \mathrm{O}_{5}, \mathrm{~K}_{2} \mathrm{O} \mathrm{ha}^{-1}$. The plants were sampled at $75,112,150$, and 225 days after sowing. We evaluated N, P and K concentrations in each of the organs (root, stem, leaf and fruit) by using wet weight, dry weight and leaf area. Our results showed that treatments with higher doses of fertilizer presented the best performance in dry matter, and they were significantly higher to those produced in treatment 2 and 1 . It was also found that the most accumulated element was nitrogen, with $72.06 \mathrm{~kg} \mathrm{~N} \mathrm{ha}^{-1}$, potassium reached a concentration of $64.39 \mathrm{~kg} \mathrm{~K} \mathrm{ha}^{-1}$ and phosphorus was the element that was accumulated in less quantity with $14.36 \mathrm{~kg} \mathrm{Pha}^{-1}$. The greatest concentrations were obtained at 225 days after sowing.
\end{abstract}

KEYWORDS: Plukenetia volubilis, mineral nutrition, absorption, nitrogen, phosphorus, potassium. 


\section{INTRODUCCIÓN}

Es conocido que el rendimiento y la calidad de un cultivo dependen de factores internos determinados por el genotipo y otros externos como las características del clima, suelo, agua, los factores bióticos, nutrición de la planta y las buenas prácticas agrícolas que se le da al cultivo. Uno de estos factores fundamentales es la nutrición mineral, que permite el control de la producción y la calidad del mismo, de tal forma que los nutrientes deben ser aplicados de acuerdo a las exigencias de la planta, en las cantidades y en las épocas adecuadas, a fin de que estos puedan ser absorbidos y asimilados. Las plantas poseen la capacidad de tomar substancias del medio y utilizarlas para la síntesis de sus componentes o como una fuente de energía (Mengel \& Kirkby, 1987; Salazar \& Juárez, 2013).

De los elementos minerales, el nitrógeno, fósforo y potasio son los elementos provenientes del suelo que se presentan en mayor concentración en los vegetales; estos elementos participan de los procesos de crecimiento como componentes estructurales y funcionales. De entre estos, el nitrógeno es un elemento esencial para los seres vivos, pues forma parte de aminoácidos, aminoenzimas, ácidos nucleicos, clorofila y alcaloides, siendo el elemento del suelo más absorbido por las plantas en condiciones normales de cultivo. Por esta razón, también es el componente que con más asiduidad se encuentra de manera deficiente en la mayoría de los cultivos en todas las partes del mundo. El nitrógeno tiene influencia en la floración y fructificación $\mathrm{y}$, por ende, en el rendimiento del cultivo (Perdomo et al., 1994; Mengel \& Kirkby, 1987). El nitrogeno puede ser absorbido por las plantas en la forma de nitrato $\left(\mathrm{NO}_{3}\right)$ o amonio $\left(\mathrm{NH}_{4}^{+}\right)$, la forma preferencial en la absorción de este elemento, ya sea nítrica o amoniacal, difiere entre las especies vegetales (Black, 1986; Coraspe et al., 2009; Molina, 2002).

El fósforo es constituyente del ATP (Adenosin Tri Fosfato), ácidos nucleicos, fosfolípidos y ciertas enzimas, este elemento cumple una función importante en el sistema de transferencia de energía dentro de la planta. Así mismo, es esencial para el crecimiento radical, en el proceso de floración y en la formación de frutas y semillas. El fosforo es absorbido por la planta principalmente como ion ortofosfato primario $\left(\mathrm{H}_{2} \mathrm{PO}_{4}^{-}\right)$, pero también se absorbe como ion fosfato secundario $\left(\mathrm{HPO}_{4=2}\right)$ y no es reducido dentro de la planta, la absorción de esta última forma se incrementa a medida que se sube el $\mathrm{pH}$.

De otro lado, el potasio (K) juega un papel importante en la fotosíntesis, pues acelera el flujo y translocación de los productos asimilados, tales como los azúcares y almidones que son formados durante la fotosíntesis y luego transportados desde las hojas hasta los órganos de reserva (frutos, semillas, tubérculos, etc). Asimismo, el potasio incrementa el rendimiento y calidad de la cosecha, mejorando el sabor, el contenido de azúcares y el color de los frutos. Favorece la resistencia a enfermedades al fortalecer los tejidos vegetativos; el potasio también mejora las propiedades de almacenamiento post cosecha de frutas y hortalizas, al promover mayor firmeza y resistencia de los tejidos (Medina et al., 1999; Molina, 2002; Rodríguez, 1992).

El sacha inchi (Plukenetia volubilis L.), es un cultivo promisorio de la Amazonía que gracias a las excepcionales propiedades nutricionales de sus semillas ha adquirido una importancia económica significativa para la industria aceitera a nivel local e internacional. A pesar de la creciente importancia económica de esta especie, algunos aspectos de su cultivo, son todavía poco conocidos, existiendo aun vacíos de información en cuanto a sus requerimientos nutricionales, para lo cual se plantea como objetivo del presente estudio analizar la fenología y determinar la absorción y concentración de N, P y K en sacha inchi (Plukenetia volubilis L.) en suelos ácidos en la Región San Martín, Perú. Se espera que los datos obtenidos sean el punto de partida para generar más información que se traduzca en beneficios a la cadena productiva de este recurso.

\section{MATERIALES Y MÉTODOS}

El estudio fue realizado en el centro experimental del Instituto de Investigaciones de la Amazonia Peruana - San Martín (IIAP-SM), 06 31' $\mathrm{S}, 7^{\circ} 17^{\prime} \mathrm{O}$ y $320 \mathrm{msnm}$, ubicado en el Centro Poblado Bello Horizonte, Distrito Banda de Shilcayo, Provincia de San Martin, Región San Martin. El estudio se realizó en un suelo con textura franco arenoso ( $\mathrm{Fr} \mathrm{A})$, de reacción muy fuertemente ácida ( $\mathrm{pH}$ : 4.71), nivel de materia orgánica fue bajo (MO: 0.97\%), bajo en fosforo disponible (P: 6.6 $\mathrm{ppm})$, bajo en potasio disponible (K: $45 \mathrm{ppm})$. Los tratamientos probados fueron tres dosis de fertilización de $\mathrm{N}, \mathrm{P}_{2} \mathrm{O}_{5}, \mathrm{~K}_{2} \mathrm{O}: \mathrm{T}_{1}: 16-23-21 \mathrm{~kg} \mathrm{~N}$, $\mathrm{P}_{2} \mathrm{O}_{5}, \mathrm{~K}_{2} \mathrm{O}$ ha $^{-1}, \mathrm{~T}_{2}: 8-12-11 \mathrm{~kg} \mathrm{~N}, \mathrm{P}_{2} \mathrm{O}_{5}, \mathrm{~K}_{2} \mathrm{O}^{-1} \mathrm{ha}^{-1} \mathrm{y}$ $\mathrm{T}_{3}: 23-35-30 \mathrm{~kg} \mathrm{~N}, \mathrm{P}_{2} \mathrm{O}_{5}, \mathrm{~K}_{2} \mathrm{O} \mathrm{ha}^{-1}$.

La plantas se muestrearon a los $75-112-150 \mathrm{y}$ 225 días después de la siembra (dds), separando la raíz, hojas, tallos y frutos, siguiendo la metodología propuestas por Bertsch, (1993) y Sancho, (1999). El número de plantas muestreadas por cada tratamiento 
fueron 12; las mismas que fueron lavadas, registrándose el peso fresco total, a continuación se tomó una muestra que fue colocada en estufa de aire forzado a $70^{\circ} \mathrm{C}$ hasta obtener un peso constante, registrándose dichos pesos para cada una de las fracciones. Estas muestras fueron molidas, embolsadas y etiquetadas y enviadas al laboratorio de Análisis de Suelos y Tejidos de la Universidad Nacional Agraria La Molina para el análisis del contenido de N, P y K en cada una de las fracciones. El $\mathrm{N}$ se determinó mediante el método de Kjeldahl, el P mediante el método de Aminonaftol Sulfónico y el K por absorción atómica (Chapman \& Pratt, 1979; Prevel et al., 1984).

El área foliar (AF) se determinó con la ayuda de papel milimetrado, colocando la hoja fresca sobre el papel, relacionando la superficie que ocupa la hoja en el papel con su peso fresco $\left(\mathrm{cm}^{2} \mathrm{~g}^{-1}\right)$. La superficie total de la hoja se obtuvo multiplicando el área foliar específica por el peso fresco total de hoja.

El diseño estadístico empleado fue el Diseño de Bloques Completamente Aleatorio-DBCA, constituido por 4 bloques y 3 tratamientos (SAS Inc., 2008).

\section{RESULTADOS Y DISCUSIÓN}

\section{Fenología del cultivo}

En general, hay una relación directa entre el crecimiento del cultivo y la producción tanto en área foliar (AF) y/o materia seca (MS); el inicio de guiado empieza a los 75 días después de la siembra (dds), la floración se inicia a los $112 \mathrm{dds}$, la fructificación a los 120 dds, el llenado de frutos a los 150 dds y la primera cosecha se inicia a los $225 \mathrm{dds}$. La definición clara de la duración y los cambios en peso de cada una de las etapas fenológicas y su relación con los cambios en las concentraciones de elementos en los diferentes tejidos de la planta, permitirá familiarizarse con los requisitos nutricionales del cultivo. La demanda de un nutrimento por un cultivo está dada por su producción de biomasa y la concentración del nutrimento en la planta o requerimiento interno. $\mathrm{Al}$ crecer, la planta desarrolla área foliar, aumenta la cantidad de tejidos, sintetiza proteínas y enzimas $\mathrm{y}$, a la vez, crea mayor número de sitios de transporte, estimulando la absorción de nutrientes (Bertsch, 1993; Greenwood, 1983; Medina et al., 1999).

En la Tabla 1, se evidencia que la materia seca y el área foliar obtenidas con el tratamiento $T_{3}$ fue estadísticamente superior a los tratamientos $\mathrm{T}_{2} \mathrm{y} \mathrm{T}_{1}$. Los rendimientos en materia seca y área foliar fueron mayores con la edad. Esto se explica debido a que la planta necesita nutrirse todos los días, absorbiendo iones de la solución suelo. Los fertilizantes utilizados por los productores son solubles y por tanto los nutrientes van directamente a la solución suelo en donde están disponibles para que la planta pueda absorberlos. Todo esto dependerá de la concentración de los elementos en la solución (a mayor concentración mayor absorción), propiedades y características del suelo y de las condiciones medioambientales.

Elementos como el N, P y K presentan una función estructural, de manera que al incrementarse hasta compensar sus requerimientos, permiten un mejor crecimiento y desarrollo de la planta (Perdomo et al., 1994; Kaiser \& Huber, 1994; Medina et al., 1999)

Tabla 1. Efecto de la fertilización de $\mathrm{N}_{1} \mathrm{P}_{2} \mathrm{O}_{5}, \mathrm{~K}_{2} \mathrm{O}$ en la producción de materia seca ( $\mathrm{MS} \mathrm{kg} \mathrm{ha}{ }^{-1}$ ) y área foliar ( $\mathrm{AF} \mathrm{m}^{2}$ ha $\left.{ }^{1}\right)$ en el cultivo de sacha inchi (Plukenetia volubilis L.).

\begin{tabular}{|c|c|c|c|c|c|c|c|c|}
\hline \multirow{3}{*}{$\underset{* *}{\text { Tratamientos }}$} & \multicolumn{8}{|c|}{ DDS* } \\
\hline & \multicolumn{2}{|c|}{75} & \multicolumn{2}{|c|}{122} & \multicolumn{2}{|c|}{150} & \multicolumn{2}{|c|}{225} \\
\hline & AF & MS & $\mathrm{AF}$ & MS & AF & MS & AF & MS \\
\hline \multirow[t]{2}{*}{$\mathrm{T}_{1}$} & 259.75 & 15.14 & 1417.73 & 257.58 & 5425.33 & 1412.37 & 13830.47 & 3213.58 \\
\hline & $b$ & $b$ & c & b & b & b & $b$ & $b$ \\
\hline \multirow[t]{2}{*}{$\mathrm{T}_{2}$} & 349.94 & 18.53 & 1850.37 & 269.21 & 4718.65 & 1393.27 & 11843.65 & 3061.27 \\
\hline & a & $\mathrm{a}$ & a & $b$ & c & c & c & c \\
\hline \multirow[t]{2}{*}{$\mathrm{T}_{3}$} & 248.08 & 15.60 & 1784.42 & 322.08 & 8538.02 & 2561.98 & 20014.02 & 4573.20 \\
\hline & $\mathrm{b}$ & $\mathrm{b}$ & $b$ & a & $\mathrm{a}$ & $\mathrm{a}$ & $\mathrm{a}$ & a \\
\hline
\end{tabular}

* Medias con diferentes letras en una misma columna difieren estadísticamente según la prueba de Duncan $(p<0.05)$.

* Días después de la siembra

* Dosis de fertilización de $\mathrm{N}, \mathrm{P}_{2} \mathrm{O}_{5}, \mathrm{~K}_{2} \mathrm{O}\left(\mathrm{kg} \mathrm{ha}^{-1}\right)$. 


\section{Absorción y concentración de N, P y K}

En general el nitrógeno, fósforo y potasio fueron absorbidos continuamente desde los 75 a 225 dds. Esto se evidencia a través de los análisis de la absorción y los cálculos de concentración y acumulación de N, P, K (Figura 1), permitiendo construir las curvas de absorción para cada elemento extraído por la planta durante su ciclo de vida (Sancho, 1999). Con esta información es posible conocer las épocas de mayor absorción de cada nutrimento y definir un programa de fertilización adecuado para el cultivo, en el cual se considere tanto la cantidad de fertilizante como la época idónea para hacer las aplicaciones.

En la Figura 1 se observa la dinámica de absorción, concentración y acumulación total por la planta de los tres elementos en estudio y, en ella, se evidencia que a los 225 días después de la siembra se registró la máxima concentración de $\mathrm{N}, \mathrm{P}$ y K en el cultivo, con los siguientes valores: $72.06 \mathrm{~kg} \mathrm{~N} \mathrm{ha}^{-1}$, $64.39 \mathrm{~kg} \mathrm{P} \mathrm{ha}^{-1}$ y $14.36 \mathrm{~kg} \mathrm{~K} \mathrm{ha}^{-1}$. Los resultados evidencian lo mencionado por Mengel \& Kirkby (1987) quienes señalan que el factor que controla el contenido nutricional del material vegetal es el potencial de absorción de los diferentes nutrientes minerales, que es específico de cada especie y determinado genéticamente. Esto explica el hecho de que el contenido de $\mathrm{N}$ y K del material de las plantas sea superior que el de P. Hay que diferenciar muy bien el movimiento de los elementos, tanto en el suelo como dentro de la planta.

En el suelo, el movimiento de los iones hacia el sistema radicular es muy diferente. Hay algunos que se mueven muy rápido por flujo de masas junto con el agua, otros se mueven muy lentamente por difusión y otros dependen del desarrollo radicular (intercepción radicular). Por otro lado, los elementos en los suelos son retenidos con diferente fuerza en el complejo de cambio, depende de las características del complejo coloidal, de las interacciones iónicas y de las características de los elementos, ya que los cationes trivalentes serán más fuertemente retenidos que los divalentes y estos más que los monovalentes. Además, depende también del grado de hidratación, pues, a menor grado de hidratación mayor retención (Mengel \& Kirkby, 1987).

En la planta, la absorción puede darse vía activa con gasto energético a través de la respiración o por vía pasiva sin gasto energético, la misma que no depende de la actividad fisiológica del vegetal. El contenido mineral de las plantas también depende con la edad del tejido. Las plantas y los tejidos más

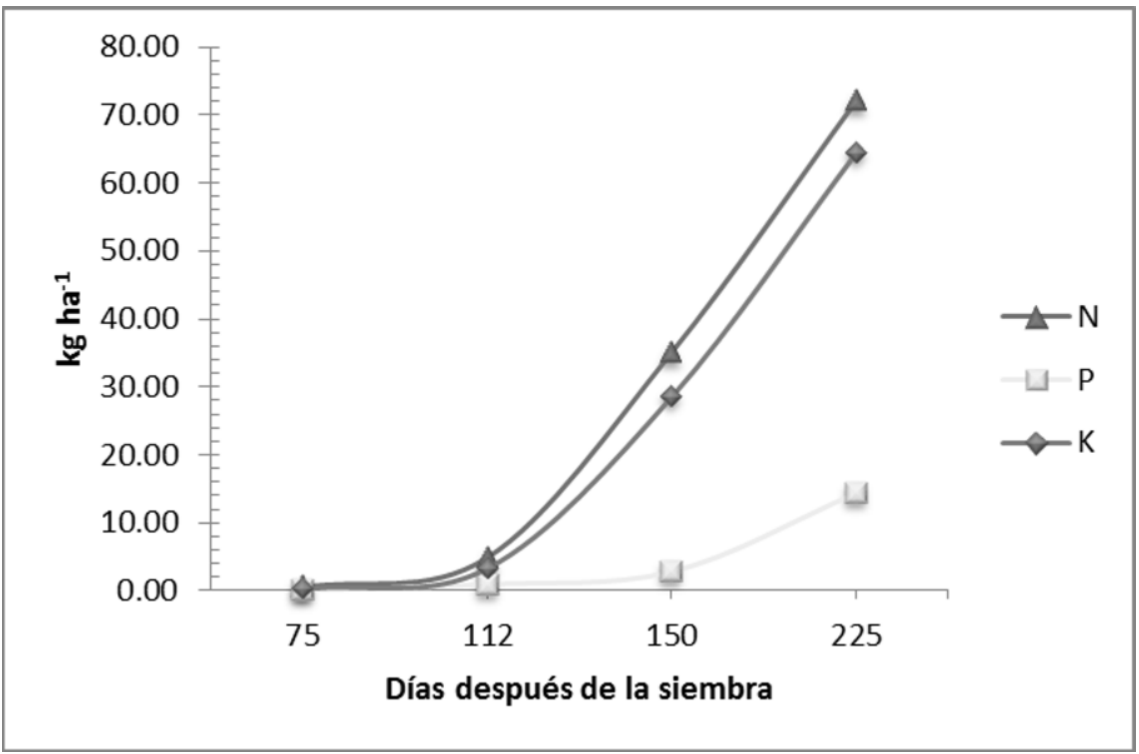

Figura 1. Concentraciones promedios de nitrógeno, fósforo y Potasio en P. volubilis a través del tiempo. 


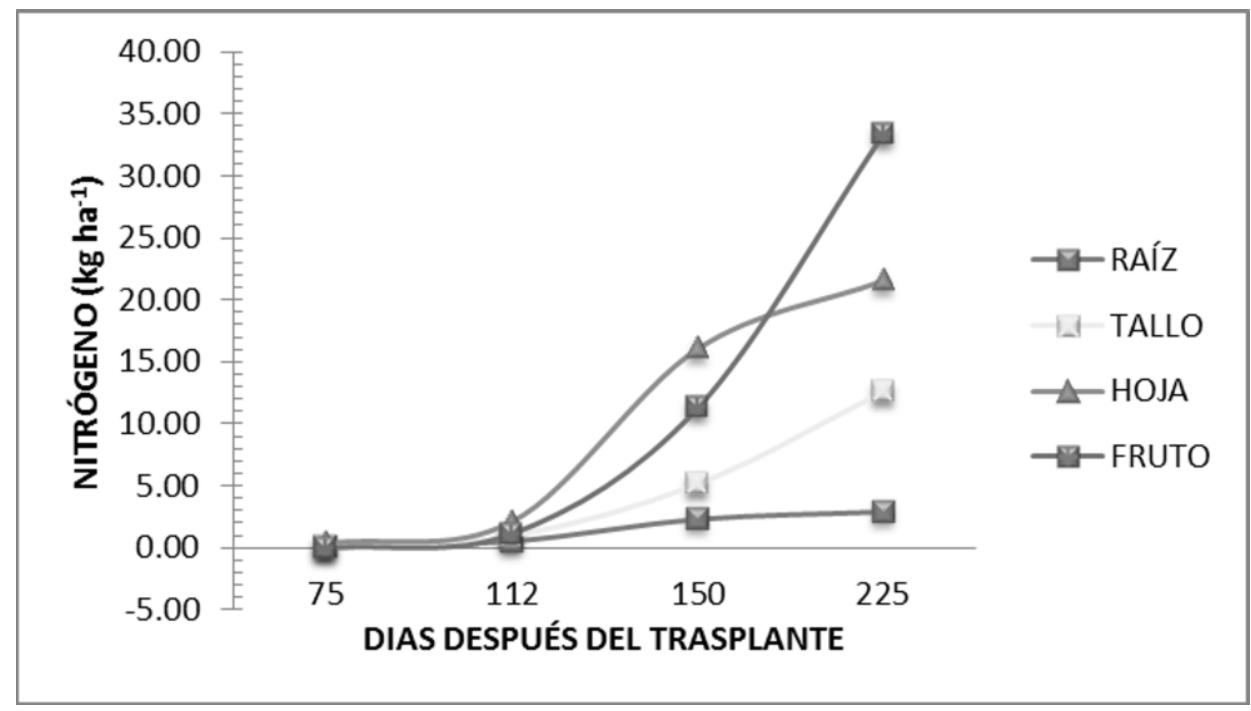

Figura 2. Concentración de Nitrógeno en la raíz, tallo, hojas y frutos de P. volubilis a través del tiempo.

jóvenes contienen más N, P y K. Salas (2002), sostiene que para cada nutrimento, el patrón de distribución, la proporción y extensión del reciclaje y removilización varía enormemente con el nutrimento, las condiciones ambientales, el estado nutricional de la planta, la especie y el estado de desarrollo. En especies anuales, la partición y removilización de nutrimentos está muy relacionado con los rápidos cambios en el desarrollo que ocurren durante el ciclo de vida de la planta. En especies perennes la absorción, partición, almacenamiento y movilización también están muy relacionados con los diferentes estados fenológicos de la planta. Esta masiva movilización de nutrimentos en las plantas nos indica que partición y movilización juegan un papel muy importante en la relación entre concentración de nutrimentos y crecimiento.

Por otro lado, también se menciona que los nutrimentos depositados en tejidos y órganos pueden ser removilizados y transportados a otras partes de la planta. La removilización en la planta de partes maduras a jóvenes durante el desarrollo o en situaciones de estrés, produce cambios en la concentración de nutrimentos, en particular en las hojas, impactando fuertemente la expresión del síntoma de deficiencia y la relación entre concentración de nutrimento con crecimiento y rendimiento de la planta.

\section{Nitrógeno}

El nitrógeno puede absorberse como $\mathrm{NO}_{3}$ y como $\mathrm{NH}_{4}{ }^{+}$, dependiendo de la preferencia de la planta. En la Figura 2, se presentan los valores de nitrógeno acumulados a través del tiempo en los distintos órganos de $P$. volubilis. En los primeros 75 días de crecimiento y desarrollo, el total de nitrógeno fue constante, debido a que la cantidad de biomasa de cada órgano de la planta fue poca; a partir de este día la acumulación de este elemento fue aumentando considerablemente en el fruto y hoja (33.35 y 21.54 $\mathrm{kg} \mathrm{Nha}{ }^{-1}$ respectivamente).

La raíz fue el órgano que acumuló menos nitrógeno (2.90 $\left.\mathrm{kg} \mathrm{N} \mathrm{ha}^{-1}\right)$, debido a que existía menor biomasa y el nitrógeno se movilizó a la parte aérea. Sin olvidar la intensa demanda fisiológica impuesta por las hojas y los tallos sobre los minerales esenciales, ni la importancia de los procesos de distribución de los mismos por parte de los tejidos vasculares, se puede decir que el proceso de nutrición mineral es fundamentalmente responsabilidad de los sistemas radicales de las plantas. En un primer momento las raíces funcionan como órganos de reserva, posteriormente son los órganos involucrados en la absorción de agua y minerales los que realizan esta función. Al igual que los tallos, las raíces pueden constituir un importante 


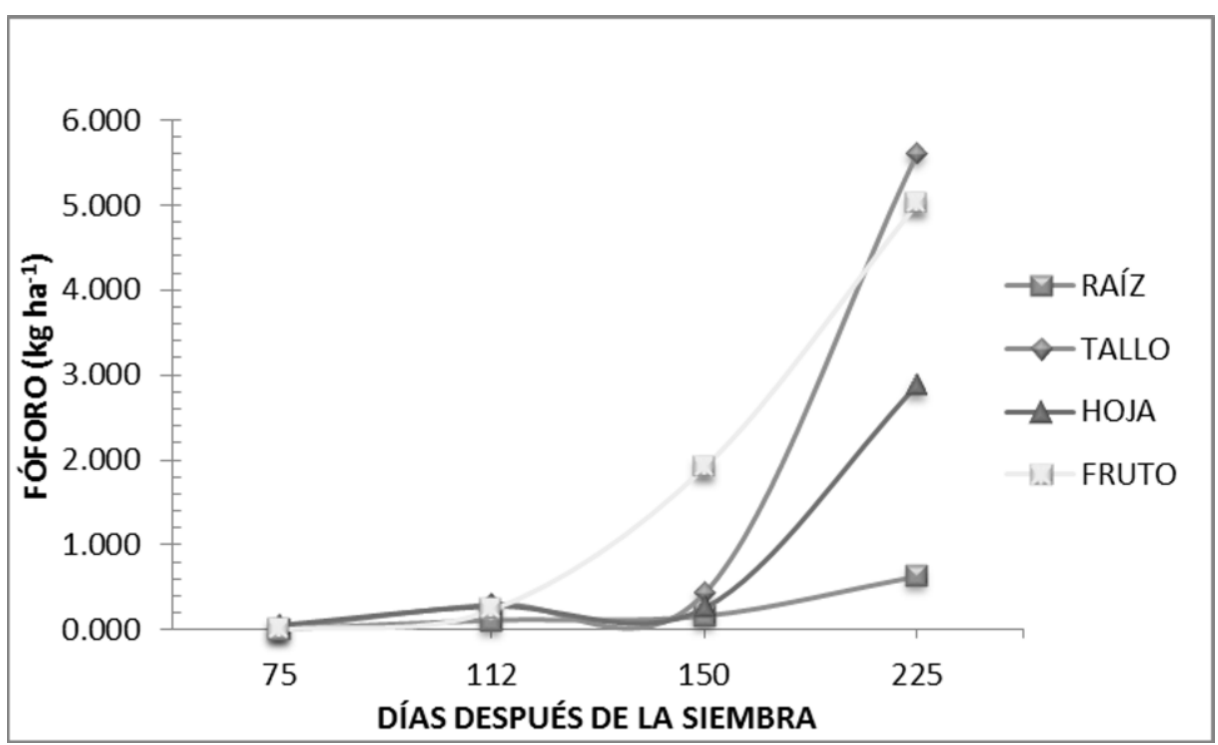

Figura 3. Concentración de Fósforo en la raíz, tallo, hojas y frutos de $P$. volubilis a través del tiempo.

órgano para el almacenamiento de agua, minerales y carbohidratos (Vinicio, 2002). La cantidad de nutrimentos en la planta depende de los procesos que se llevan a cabo en el suelo, lo que implica que cuando la disponibilidad excede a la demanda, varios procesos actúan para evitar dicho exceso (Salas, 2002). En el tallo se registró una acumulación de $12.58 \mathrm{~kg} \mathrm{~N} \mathrm{ha}^{-1}$, esto se explica debido a que los tallos constituyen la vía para el tráfico de minerales a larga distancia dentro de las plantas, tanto en el xilema como en el floema, de la raíz al follaje y viceversa (Vinicio, 2002). El movimiento de nutrimentos en la planta depende de la capacidad de absorción y de la demanda del nutrimento (Salas, 2002).

A los 150 dds, la hoja fue el órgano en el que más nitrógeno se acumuló (16.04 $\mathrm{kg} \mathrm{N} \mathrm{ha}^{-1}$ ); a los 225 dds, registró una acumulación $21.54 \mathrm{~kg} \mathrm{~N} \mathrm{ha}^{-1}$, lo cual fue superado por el fruto. Esto demuestra que las hojas pasan de ser almacenes de nutrientes a fuentes de estos minerales, ya que estos se desplazan a los órganos de reserva que en este caso vendría a ser el fruto. Vinicio (2002) sostiene que la demanda de nutrimentos por parte de las hojas cambia durante el ciclo de vida y muestra una relación estrecha con la tasa y las características del crecimiento. La longevidad de las hojas está fuertemente determinada por el estado fisiológico de las plantas en el momento de su producción. Lo mismo explican Mengel \& Kirkby (1987), quienes mencionan que cuando el suministro de nitrógeno desde el medio edáfico es inadecuado, el $\mathrm{N}$ de las hojas se moviliza para alimentar a los órganos más jóvenes de la planta. El fruto constituye el órgano de almacenamiento de nutrientes, pues superó a los demás órganos en la concentración de nitrógeno (33.35 $\left.\mathrm{kg} \mathrm{N} \mathrm{ha}^{-1}\right)$ a los 225 dds. Vinicio (2002) sostiene que, el cuaje de los frutos es en gran medida dependiente de la actividad de las semillas que ellos mismos contienen. El posterior desarrollo de los frutos hasta culminar en la maduración exitosa, constituye otra historia tan compleja como la del desarrollo de las flores. Ambos dependen de múltiples factores ambientales y fisiológicos, tanto presentes (condiciones climáticas actuales), como pasados (nutrición mineral previa, historia fenológica).

\section{Fósforo}

En la Figura 3, se muestra el contenido de fósforo acumulado a través del tiempo en $P$. volubilis. A los 225 dds, el órgano con menor concentración fue la raíz con $0.63 \mathrm{~kg} \mathrm{P} \mathrm{ha}^{-1}$; el tallo y el fruto fueron los órganos en el que más fósforo se acumuló $(5.60 \mathrm{y}$ $5.01 \mathrm{~kg} \mathrm{P} \mathrm{ha}^{-1}$ respectivamente). En las hojas la concentración fue $2.88 \mathrm{~kg} \mathrm{P} \mathrm{ha}^{-1}$. A pesar que las raíces son los órganos involucrados en la absorción de agua y minerales por excelencia, aún hay duda si los compuestos orgánicos de $\mathrm{P}$ son absorbidos por las raíces de las plantas en grandes cantidades (Mengel \& Kirkny, 1987), lo que concuerda con la 
baja concentración de fosforo en las raíces y por ende en la planta. Sus atributos morfológicos y fisiológicos, expresados por ejemplo en su alta relación superficie/volumen y en la plasticidad de su arquitectura, determinan su éxito ecológico en el forrajeo de nutrientes y agua en un ambiente hostil y competitivo (el suelo), donde el abastecimiento de los recursos es limitado, local y variable (Vinicio, 2002). El destino de estos minerales es variado y depende de las relaciones fruente-sumidero y de la especificidad de las funciones desempeñadas por cada mineral.

La concentración de nutrientes varía no solo entre las diferentes especies, sino también, en los distintos órganos de una misma planta. Esta variación está afectada por el tipo de tejido de planta, la edad fisiológica del tejido, la posición del tejido en la planta, la disponibilidad y concentración de minerales en el substrato, los factores climáticos y las condiciones del suelo, es importante además como los distintos órganos responden de diferente manera ante las variaciones en la concentración de nutrientes y la demanda de éstos durante la ontogénesis (Medina et al., 1999). Los tallos representan a la vez un importante consumidor de recursos minerales para sustentar la producción de tejidos vasculares y accesorios, la actividad del cambium y el crecimiento expansivo en especies perennes, y el almacenamiento de reservas (Vinicio, 2002).
El fosfato es muy móvil en la planta y es por eso que puede translocarse hacia arriba y hacia abajo. El fosfato absorbido por las células de las plantas se ve rápidamente involucrado en los procesos metabólicos. Las hojas jóvenes quedan suministradas no solo con fosfato absorbido por las raíces, sino también con fosfato procedente de hojas más maduras (Bouma, 1967; Mengel \& Kirkby, 1987).

\section{Potasio}

La Figura 4, muestra la absorción de potasio en cada órgano de $P$. volubilis, evidenciándose los cambios significativos en el tiempo. La raíz al igual que para el $\mathrm{N}$ y $\mathrm{P}$, es el órgano donde menos acumulación de $\mathrm{K}$ se registró $\left(2.81 \mathrm{~kg} \mathrm{~K} \mathrm{ha}^{-1}\right)$. El tallo y el fruto fueron los órganos donde mayor acumulación de $\mathrm{K}$ se ha registrado, (22.44 y 22.83 $\mathrm{kg} \mathrm{K} \mathrm{ha}^{-1}$ respectivamente). Las hojas registraron una acumulación de $15.17 \mathrm{~kg} \mathrm{~K} \mathrm{ha}^{-1}$.

El potasio presenta una alta velocidad de absorción por los tejidos vegetales (Mengel \& Kirkby, 1987). La distribución de los asimilados orgánicos y de los minerales en las plantas parece estar determinada por la siguiente jerarquía fisiológica: semillas, frutos carnosos, los meristemos y las hojas, el cambium, raíces y el almacenamiento (Vinicio, 2002).

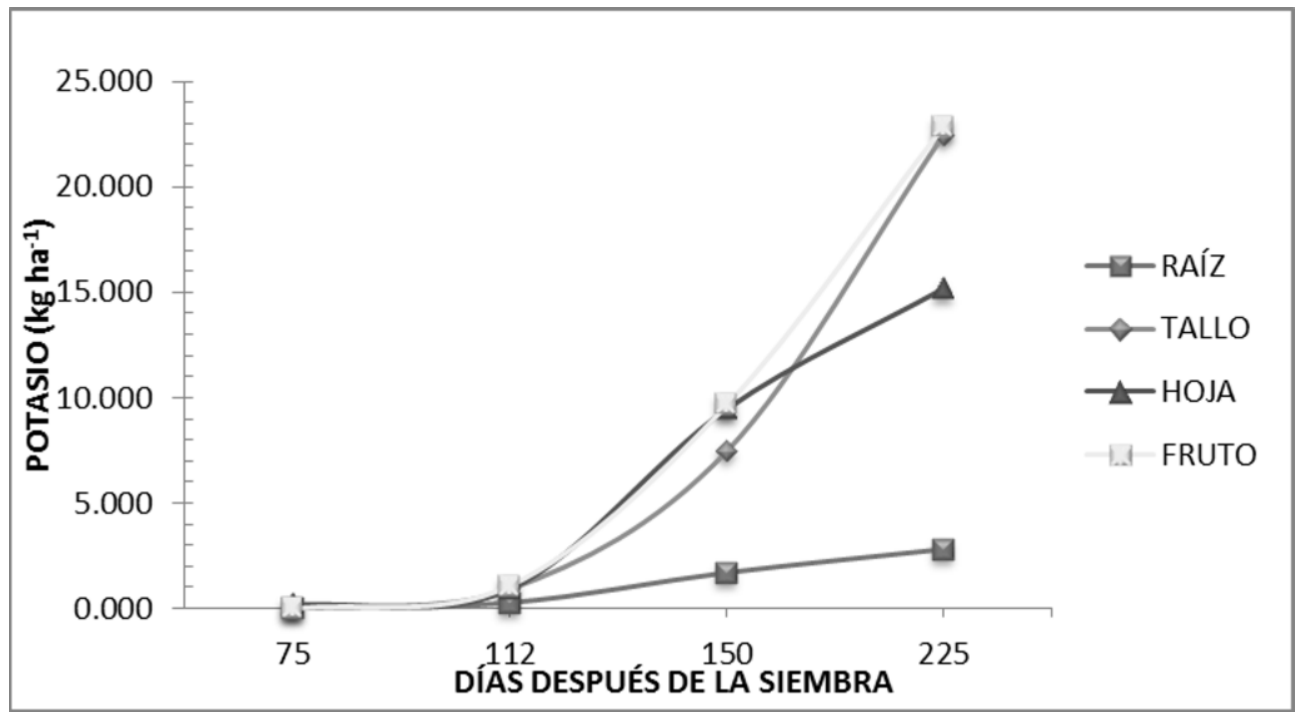

Figura 4. Concentración de Potasio en la raíz, tallo, hojas y frutos de P. volubilis a través del tiempo. 
Mengel \& Kirkby (1987), mencionan que la dirección del transporte de $\mathrm{K}$ suele ser hacia los tejidos jóvenes, dándose con frecuencia una redistribución desde las partes más viejas de la planta a las más jóvenes. El tallo puede constituir un importante órgano de reserva (particularmente en especies perennes) de agua, minerales y compuestos orgánicos, movilizables durante períodos de estrés (déficit hídrico, defoliación, podas). El crecimiento secundario de las especies perennes representa una alta demanda de minerales, necesarios para la actividad del cambium. Una proporción importante de estos minerales es inmovilizada en el duramen (xilema no conductor) de los troncos y de las raíces.

La alta velocidad de absorción de $\mathrm{K}^{+}$puede ser debido al efecto sinérgico que ejerce el $\mathrm{N}$, especialmente el N-NO ${ }_{3}^{-}$, ya que la planta al absorber $\mathrm{NO}_{3}^{-}$, este puede jalar al $\mathrm{K}^{+}$a través de fuerzas electrocinéticas (cargas positivas y negativas).

Las hojas representan uno de los principales consumidores. Sin embargo, el reciclaje (removilización) de algunos minerales, durante la senectud de las hojas o la reproducción, es muy reducido. Una proporción importante de los minerales permanece en los tejidos vegetativos no cosechados y su reincorporación puede contribuir a mejorar la fertilidad del suelo (Vinicio, 2002). La mayor parte de $\mathrm{K}$ se absorbe principalmente durante la etapa de crecimiento vegetativo (Mengel \& Kirkby, 1987).

Dado estos resultados y bajo las condiciones en que se realizó esta investigación nosotros podemos concluir que en Plukenetia volubilis el nitrógeno fue el elemento acumulado en mayor cantidad $(72.06 \mathrm{~kg}$ $\mathrm{N} \mathrm{ha}^{-1}$ ), seguido del potasio (64.39 $\mathrm{kg} \mathrm{K}^{-1}$ ) y el fósforo (14.36 kg P ha ${ }^{-1}$ ); y que la máxima acumulación de estos tres elementos se dio a los 225 días después del trasplante, coincidiendo con la época de la primera cosecha.

\section{BIBLIOGRAFÍA CITADA}

Bertsch, F. 1993. Curvas de absorción de nutrimentos en aráceas. In: Taller sobre aplicaciones de la biotecnología en raíces, tubérculos y pejibaye. CIA. UCR. San José, Costa Rica.

Black, C. 1986. Soil plant relationship. 2nd ed. New York: John Wiley. 405 pp.

Bouma, D. 1967. Nutrient uptake and distribution in subterranean clover during recovery from nutritional stresses. I. Experiments with phosphorus. Aust. J. Biol. Sci. 20: 601 -613.

Chapman, H.; Pratt, P. 1979. Métodos de análisis para suelos, plantas y aguas. Ed. Trillas, México. $195 \mathrm{pp}$.

Coraspe, H.; Takashi, M.; Vinicus, I.; F. Contreras; Ocheuze, P. 2009. Absorción de Macronutrientes por plantas de Papa (Solanumtuberosum L.) en La Producción de Tubérculo-Semilla. Agronomía Tropical, 59(1): 45-58.
Greenwood, D. 1983. Quantitative theory and the control of soil fertility. New Phytol, 94: 1-18.

Kaiser, W.; Huber, S. 1994. Modulation of nitrate reductase in vivo and in vitro: Effects of phosphoprotein phosphatase inhibitors, free $\mathrm{Mg}^{2+}$ and 5' AMP. Planta 193, 358-364.

Medina, G.; Orozco, M.; Bolívar, J.; Ramírez, P. 1999. Acumulación y concentración de nitrógeno, fósforo y potasio en Gypsophila paniculata L. cv. Perfecta. Agronomía Colombiana, 16(1-3): 46-60.

Mengel, K.; Kirkby, E. 1987. Principios de nutrición vegetal. 4a. Edición. 692 pp.

Molina, E. 2002. Fuentes de fertilizantes foliares. Seminario de Fertilización foliar: Principios y Aplicaciones. Laboratorio de Suelos y Foliares en colaboración con la Asociación Costarricense de la Ciencia del Suelo. Costa Rica. p. 26-35.

Perdomo, C.; Barbazán, M.; Duran, J. 1994. Nitrógeno. Cátedra de Fertilidad. Facultad de Agronomía. Universidad de la Republica. Montevideo. Uruguay, 74 pp.

Prevel, M.; Gacnard, J.; Gautier, P. ; Drouinau G. 1984. L'Analyse Végétale dans le control de l'Alimentation des Plantes temeperées y tropical. Technique et Documentation - Lavoisier. París. 161-179 pp.

Rodríguez, S. 1992. Fertilizantes, nutrición vegetal. AGT 1era edición. Segunda reimpresión. México, D.F. 124 pp.

Salas, R. 2002. Herramientas de diagnóstico para definir recomendaciones de fertilización foliar. Seminario de Fertilización foliar: Principios y Aplicaciones. Laboratorio de Suelos y Foliares en colaboración con la Asociación Costarricense de la Ciencia del Suelo. Costa Rica. p. 7 - 18.

Salazar, F.; Juárez, P. 2013. Requerimiento macronutrimental en las plantas de chile (Capsicum annuum L.). Biociencias, 2 (2): 27 34

Sancho, H. 1999. Curvas de absorción de nutrientes: importancia y uso en los programas de fertilización. Instituto de la Potasa y el Fósforo. Informaciones Agronómicas, 36:11-13.

SAS Institute Inc. 2008. SAS/STAT 9.2 User's Guide, Ch. 7. SAS Institute, Inc., Cary, NC. 179 pp.

Vinicio, F. 2002. Aspectos Mecanismos de absorción de nutrimentos por el follaje. Seminario de Fertilización foliar: Principios y Aplicaciones. Laboratorio de Suelos y Foliares en colaboración con la Asociación Costarricense de la Ciencia del Suelo. Costa Rica.p. 1 - 6.

Recibido: 18 de julio del 2015

Aceptado para publicación: 25 de agosto del 2015 